\title{
Aerobic bacteria from oral cavities and cloaca of snakes in a petting zoo
}

\author{
Yeon-Sook Jho ${ }^{1, \uparrow}$, Dae-Hun Park ${ }^{2, \uparrow}$, Jong-Hwa Lee ${ }^{2}$, Young S. Lyoo ${ }^{3, *}$ \\ ${ }^{1}$ Snoopy Animal Hospital, Gunpo 435-040, Korea \\ ${ }^{2}$ Toxicology Center, Korea Institute of Toxicology, Daejeon 305-343, Korea \\ ${ }^{3}$ College of Veterinary Medicine, Konkuk University, Seoul 143-701, Korea \\ (Received: April 25, 2011; Revised: June 06, 2011; Accepted: June 07, 2011)
}

\begin{abstract}
It is important to identify the bacteria in snakes because they can cause disease; importantly, bacteria such as Stenotrophomonas maltophilia, Escherichia coli, Proteus vulgaris etc. could be pathogens especially in hospitalized, debilitated hosts, and immunocompromised patients. To analyze the distribution of snakes' bacteria in petting zoo, samples from 20 snakes were collected from 2002 to 2008. Nine bacteria species were isolated from both oral and cloaca while four and six species were identified only from oral and cloaca, respectively. Except for Actinobacter sp., all of the identified strains are opportunistic pathogens, and most of them can cause nosocomial infections in humans. Present results indicate that prevalence of various zoonotic bacterial strains in snakes could be involved in potential transfer of these bacteria into caretakers and other animals. Therefore, it needs to examine the antibiotic resistance of these pathogens to prevent outbreaks.
\end{abstract}

Keywords : bacteria, opportunistic infection, petting zoo, snake, zoonosis

As society develops, the chance that humans have contact with animals decreases; however, the desire of people interact with animals increases. As a result, the number of zoos where people can observe and often interact with wildlife has risen. Snakes are among the wild animals that can often be handled in zoos. However, even though microorganism-induced diseases can dramatically affect snake health, there have not been many studies on the distribution of bacteria in snakes and how the environment can affect the predominant strains of bacteria. For example, under healthy conditions, the predominant oral florae of snakes are gram-positive bacteria such as Corynebacterium and Staphylococcus. However, when snakes are affected by stomatitis, the predominant oral bacteria are gram-negative such as Pseudomonas aeruginosa, Providencia rettgeri, and Pseudomonas maltophilia. These bacteria are not exogenic pathogens, but rather opportunistic invaders [10].

Reptiles can often act as reservoirs for microorganismmediated diseases in humans and animals. In snakes, bacteria such as Staphylococcus spp., Pseudomonas spp.,

\footnotetext{
*Corresponding author

Tel: +82-2-450-3719, Fax: +82-2-6008-3791

E-mail: lyoo@konkuk.ac.kr

†The first two authors equally contributed to this study.
}

Salmonella arizonae, and Enterobacteriaceae are normal florae. When snakes are infected with microorganisms such as Acinetobacter calcoaceticus var. anitratus, Hafnia alvei, Arizona hinshawii, Shigella spp., Klebsiella oxytoca, and Pseudomonas aeruginosa, they have the potential to transmit these infections to humans [2]. Aeromonas hydrophilia infections are transferred by snake-bites and can induce gastroenteritis, soft tissue infections, and pneumonia [11]. Enterococcus faecalis is normally found in the gastrointestinal (GI) tract of snakes, but during pathological conditions this pathogen can gain resistance towards antibiotics [6]. Enterobacter is a Gram-negative, rod-shaped bacterium, some strains of which can cause opportunistic infections, most commonly in the urinary and respiratory tracts [10]. Proteus $s p$. is a pathogen that can cause urinary tract diseases and often causes nosocomial outbreaks [5]. Pseudomonas $s p$. is an opportunistic pathogen that can lead to the breakdown of non-specific host defenses [10]. Although Stenotrophomonas maltophilia is widely distributed in the environment, it can be an opportunistic 
Table 1. Common and Scientific Names of Experimental Snakes

\begin{tabular}{llc}
\hline \multicolumn{1}{c}{ Common name } & \multicolumn{1}{c}{ Scientific name } & Heads \\
\hline Albino Burmese python & Python molurus bivittatus & 3 \\
Ball Python & Python regius & 1 \\
Burmese python & Python molurus bivittatus & 5 \\
California Banded King Snake & Lampropeltis getula & 1 \\
Corn Snake & Elaphe guttata & 1 \\
King Snake & Lampropeltis getula & 1 \\
Red-tailed Boa & Boa Constrictor & 2 \\
Reticulated Python & Python reticulatus & 3 \\
Yellow Anaconda & Eunecres notaeus & 1 \\
Yellow Mangrove Snake & Boiga dendrophila & 2 \\
Sum & & 20 \\
\hline
\end{tabular}

pathogen and infect humans, as it is associated with infections like endocarditis, sepsis, meningitis, peritonitis, and soft tissue and wound infections [16].

In Korea, there are few data on the bacteria of snakes, but the possibility of transferring these infections to humans via snakes has recently increased due to petting zoos. The risk to those who routinely handle and care for the snakes is greatly enhanced. The purposes of this study were to characterize the bacteria of snakes in petting zoos in Korea, identify the aerobic bacteria with the potential to induce zoonosis, and educate the public to decrease the risk of transferring these infections between snakes and humans.

Samples for bacteria culture were collected from 20 snakes that were exhibited from January 2002 to December 2008 in a Korean petting zoo located at 126.93 degrees east longitude and 37.5 degrees north latitude (Table 1). After clinical signs were observed, the animals that appeared to be in good health were selected for the study. Animals were immobilized during sample collection. To obtain oral samples, a sterile transport swab with liquid Amies medium (Copan Innovation, Italy) was introduced between the larynx and the bottom of the inner gum line. Cloaca samples were similarly isolated. Samples were inoculated on culture plates with blood agar (Becton, Dickinson and company, USA) and incubated at $37^{\circ} \mathrm{C}$ for $18 \mathrm{~h}$.

Isolated colonies were examined using Gram staining, microscopic observations, oxidase tests, and catalase tests. Gram negative bacterial samples were analyzed using an API 20NE System (bioMerieux, France) and Gram positive bacterial samples were analyzed using a BBL Crystal Identification System (Becton, Dickinson and company, USA). To confirm these results, the
Table 2. Bacterial Strains in Oral and/or Cloaca Samples

\begin{tabular}{lcc}
\hline \hline & Oral & Cloaca \\
\hline Actinobacter sp. & 4 & 4 \\
Aeromonas hydrophillia & 1 & 1 \\
Citrobacter sp. & 4 & 3 \\
Enterobacter sp. & 2 & 4 \\
Escherichia coli & 7 & 7 \\
Morganella morganii & 1 & 1 \\
Proteus sp. & 7 & 9 \\
Pseudomonas sp. & 10 & 1 \\
Stenotrophomonas maltophilia & 4 & 1 \\
Bacillus thuringiensis & 1 & - \\
Providencia sp. & 2 & - \\
Serratia marcescens & 2 & - \\
Staphylococcus auerus & 1 & - \\
Corynebacterium pseudogenitalium & - & 2 \\
Enterococcus faecalis & - & 4 \\
Klebsiella pneumonia & - & 6 \\
Micrococcus roseus & - & 1 \\
Salmonella arizonae & - & 2 \\
Staphylococcus lentus & - & 2 \\
. Proteus mirabilis. & &
\end{tabular}

*Proteus mirabilis: Oral-2, Cloaca-4; Proteus vulgaris: Oral -5 , Cloaca-5.

isolated colonies were reanalyzed using a VITEK I System (bioMérieux, USA) from January 2002 to April 2007 and a VITEK II System (bioMérieux, USA) from May 2007 to December 2008.

Nine bacterial strains, including Actinobacter sp., Aeromonas hydrophillia, Citrobacter sp., Enterobacter sp., Escherichia coli, Morganella morganii, Proteus sp., Stenotrophomonas maltophilia, and Pseudomonas sp. were isolated from both oral and cloaca samples. 
The incidence rates of Escherichia coli and Proteus $s p$. $(35 \%)$ in both oral and cloaca cultures were higher than that of the other strains. The incidence rates of Stenotrophomonas maltophilia and Pseudomonas $s p$. in the oral samples (20 and $50 \%$, respectively) were much higher than in the cloaca samples (5\% in both).

Bacillus thuringiensis, Providencia sp., Serratia marcescens, and Staphylococcus auerus were isolated only from oral samples with incident rates of 5, 10, 10, and $5 \%$, respectively.

Corynebacterium pseudogenitalium, Enterococcus faecalis, Klebsiella pneumonia, Micrococcus roseus, Salmonella arizonae, and Staphylococcus lentus were isolated from cloaca samples with incident rates of 20 , 10 , and $10 \%$, respectively.

Zoonotic bacteria with incidence rates over $20 \%$ (4 or more of the 20 snakes) included Citrobacter sp., Enterobacter sp., Escherichia coli, Proteus sp., Stenotrophomonas maltophilia, Pseudomonas sp., and Enterococcus faecalis. The incidence rate for Pseudomonas $s p$. was the highest (50\%), followed by that of Proteus $s p$. (45\%), as Pseudomonas $s p$. was isolated from oral samples from 10 snakes and in both oral and cloaca samples from one snake. Proteus $s p$. was identified in cloaca samples from nine snakes.

Oral and cloaca samples were obtained from snakes residing in Korean petting zoos to identify which bacterial strains were present and to assess the risk of transferring diseases from snakes to humans in this context. Nine strains of coincidental bacteria were isolated from oral and cloaca samples, including Actinobacter sp., Aeromonas hydrophillia, Citrobacter sp., Enterobacter sp., Escherichia coli, Morganella morganii, Proteus sp., Stenotrophomonas maltophilia, and Pseudomonas sp. Except for Actinobacter sp., all of the identified strains are opportunistic pathogens, and most of them can cause nosocomial infections in humans [10]. Though the incidence rates were low, of the four bacterial samples isolated from only oral samples, Providencia sp., Serratia marcescens, and Staphylococcus auerus can induce human disease [19, 20]. Of the six bacterial samples isolated from only cloaca samples, Enterococcus faecalis, Salmonella arizonae, and Staphylococcus lentus are zoonotic pathogens [7, 14, 18]. These results suggest that the snakes housed in one Korean petting zoo were broadly infected with various strains of zoonotic bacteria that can be a threat to the health of humans who come into contact with the snakes.

Snakes live in most areas of Earth except for Antarctica, and there are an estimated 8,000 species. Snakes are legless earth animals so mostly inhabit the subterranean region and they prey upon other animals on the ground. So snakes are enclosed and influenced by environmental bacteria. Therefore a wide variety of bacteria can cause generalized bacterial infections. Ulcerative or necrotic dermatitis, infectious cloacitis, mouth rot (infectious stomatitis) caused by traumatic injury, bite wounds, or poor environmental quality are seen in all orders of reptiles. The aerobes commonly isolated from infected snakes are Pseudomonas, Aeromonas, Serratia, Salmonella, Micrococcus, Proteus, Staphylococcus, Streptococcus, Escherichia coli, etc.

Signs of mouth rot can vary in number and degree, depending on the stage of infection. Anorexia, purulent discharge (pus) in the mouth, excessive mucous (salivation) in the mouth, swelling or reddening around or in the mouth, inability to close the mouth, reduced or absent tongue flicking, and gingival swelling or necrosis may all be seen. The lining of the mouth can become eroded and often develops a "cottage cheese" appearance that is either yellow- or whitish-gray in color.

In extremely advanced cases, the head may be swollen; the infection may spread to the jaw and/or cranium (skull); teeth may become loose; and pneumonia may develop from aspiration of bacteria. Bacteria may also travel up what is called the hardarian duct, in this case, the eye may become infected and swell. The intestinal lining may become enteritis as a result of the ingestion of necrotic material that has broken off from an area of plaque in the mouth. In areas where necrosis and ulceration is especially deep, a blood clot may form that is loaded with bacteria and released into the bloodstream with a resultant septicemia.

However, various bacterial strains in the rhizosphere, such as Burkholderia, Ochrobactrum, and Stenotrophomonas, can cause opportunistic human diseases [1]. Bacteria, including Streptococci spp., Staphylococcus spp., Enterococci spp., Aeromonas hydrophilia, Citrobacter spp., Enterobacter spp., Escherichia coli, Morganella morganii, Proteus spp., Pseudomonas pseudoalcaligenes, Salmonella arizonae, Serratia spp., Yersinia enterocolitica, and Bacteroides spp., can infect humans via snake bites $[12,13,15,17]$.

Stenotrophomonas maltophilia (formerly Xanthomonas maltophilia) is a non-fermenting Gram-negative bacillus, 
is widely distributed, and has an increased potential to be an opportunistic pathogen to humans during hospitalizations, malignancies, chronic respiratory diseases, and endotracheal intubation [8]. An additional threat is that Stenotrophomonas maltophilia has an intrinsic resistance to broad-spectrum antibiotics [9]. Stenotrophomonas maltophilia is associated with infections such as endocarditis, sepsis, meningitis, peritonitis, and soft tissue and wound infections [16]. Infections with Stenotrophomonas maltophilia in cystic fibrosis patients have become prevalent in recent years [3].

Due to the high incidence rates of zoonotic bacterial strains in snakes from a petting zoo, our data suggest that there should be concern about human/snake interactions in such environments. This survey is merely started to establish the database for diagnosis and treatment of reptile including wildlife. As reptile is ectodermic animals and the climate of southern Korean Peninsula is temperate so difference of bacteria cultured at temperature is worthy of notice. Therefore, there is a need for following experiment of the cultivation under various conditions and to antibiotics resistance test. It is proposed to carry out characterizing of these opportunistic bacteria to protect against an outbreak of these pathogens.

\section{References}

1. Berg G, Eberl L, Hartmann A. The rhizosphere as a reservoir for opportunistic human pathogenic bacteria. Environ Microbiol 2005, 7, 1673-1685.

2. Blaylock RS. Normal oral bacterial flora from some southern African snakes. Onderstepoort J Vet Res 2001, 68, 175-182.

3. de Vrankrijker AMM, Wolfs TFW, van der Ent CK. Challenging and emerging pathogens in cystic fibrosis. Paediat Respir Rev 2010, 11, 246-254.

4. Draper CS, Walker RD, Lawler HE. Patterns of oral bacterial infection in captive snakes. J Am Vet Med Assoc 1981, 179, 1223-1226.

5. Endimiani A, Luzzaro F, Brigante G, Perilli M, Lombardi G, Amicosante G, Rossolini GM, Toniolo A. Proteus mirabilis bloodstream infections: risk factors and treatment outcome related to the expression of extended-spectrum $\beta$-lactamases. Antimicrob Agents Chemother 2005, 49, 2598-2605.

6. Gilmore MS, Ferretti JJ. Microbiology. The thin line between gut commensal and pathogen. Science 2003,
299, 1999-2002.

7. Giridhara Upadhyaya PM, Ravikumar KL, Umapathy BL. Review of virulence factors of enterococcus: An emerging nosocomial pathogen. Indian J Med Microbiol 2009, 27, 301-305.

8. Goss CH, Otto K, Aitken ML, Rubenfeld GD. Detecting Stenotrophomonas maltophilia does not reduce survival of patients with cystic fibrosis. Am J Respir Crit Care Med 2002, 166, 356-361.

9. Hu LF, Chang X, Ye Y, Wang ZX, Shao YB, Shi $\mathbf{W}, \mathbf{L i} \mathbf{X}, \mathbf{L i}$ JB. Stenotrophomonas maltophilia resistance to trimethoprim/sulfamethoxazole mediated by acquisition of sul and $d f r A$ genes in a plasmidmediated class 1 integron. Int $\mathbf{J}$ Antimicrob Agents 2011, 37, 230-234.

10. Iglewsk BH. Pseudomonas. In: Baron S (ed.). Medical Microbiology. 4th ed. Chapter 27, University of Texas Medical Branch, Galveston, 1996.

11. Janda JM, Abbott SL. The genus Aeromonas: taxonomy, pathogenicity, and infection. Clin Microbiol Rev 2010, 23, 35-73.

12. Kerrigan KR. Bacteriology of snakebite abscess. Trop Doct 1992, 22, 158-160.

13. Liao WB, Lee CW, Tsai YS, Liu BM, Chung KJ. Influential factors affecting prognosis of snakebite patients management: Kaohsiung Chang Gung Memorial Hospital experience. Chang Gung Med J 2000, 23, 577-583.

14. Mahajan RK, Khan SA, Chandel DS, Kumar N, Hans C, Chaudhry R. Fatal case of Salmonella enterica subsp. arizonae gastroenteritis in an infant with microcephaly. J Clin Microbiol 2003, 41, 58305832.

15. Nishioka Sde A, Silveira PV. Bacteriology of abscesses complicating bites of lance-headed vipers. Ann Trop Med Parasitol 1992, 86, 89-91.

16. Sattler CA, Mason EO Jr, Kaplan SL. Nonrespiratory Stenotrophomonas maltophilia infection at a children's hospital. Clin Infect Dis 2000, 31, 1321-1330.

17. Shek KC, Tsui KL, Lam KK, Crow P, Ng KHL, Ades G, Yip KT, Grioni A, Tan KS, Lung DC, Lam TSK, Fung HT, Que TL, Kam CW. Oral bacterial flora of the Chinese cobra (Naja atra) and bamboo pit viper (Trimeresurus albolabris) in Hong Kong SAR, China. Hong Kong Med J 2009, 15, 183-190.

18. Stepanovic S, Ježek P, Vukovic D, Dakic I, Petrás P. Isolation of members of the Staphylococcus sciuri 
group from urine and their relationship to urinary tract infections. J Clin Microbiol 2003, 41, 5262-5264.

19. Svejgaard E. The role of microorganisms in atopic dermatitis. Semin Dermatol 1990, 9, 255-261.

20. Voelz A, Müller A, Gillen J, Le C, Dresbach T,
Engelhart S, Exner M, Bates CJ, Simon A. Outbreaks of Serratia marcescens in neonatal and pediatric intensive care units: clinical aspects, risk factors and management. Int $\mathbf{J}$ Hyg Environ Health 2010, 213, 79-87. 